\title{
Anti-Evolutionary Targets in Bacterial Efflux Pumps: Future Therapeutics to Combat Antibacterial Resistance
}

\author{
Pavan Gollapalli* \\ Department of PG studies and Research in Biotechnology and Bioinformatics, Kuvempu University, India \\ Submission: February 12, 2017; Published: February 28, 2017
}

"Corresponding author: Gollapalli Pavan, Department of PG studies and Research in Biotechnology and Bioinformatics, Jnana Sayhadri campus, Shankaraghatta-577451, Karnataka, India, Email: gollapalli.pavan@gmail.com

\begin{abstract}
The global emergence of multi-drug resistance in the pathogenic bacteria is a growing threat to antibiotic therapy. One of the major contributions to antibiotic resistance was by efflux mechanisms in the bacteria and they present a major challenge for antibiotic development. Hence, comprehensive understanding of the drug resistance mechanisms that are adopted by the regulation and physiological functions of efflux pumps at systems level. In this review, by attempt to present a brief outline to develop anti-evolutionary drugs to combat antibacterial resistance in most of the pathogens.
\end{abstract}

Keywords: Antibacterial resistance; Anti-evolutionary targets; Efflux pumps; Systems biology

\section{Introduction}

Over the years, it has been a significant biochemical issue to understand the mechanism of drug resistance in most of the pathogens [1]. The resistances in some species of bacteria have been unfortunately developed to a level that no clinically available treatment is effective [2]. The mechanisms involved in the resistance may be depend upon the nature of antibiotics, its target sites, the bacterial species and whether it is mediated by a resistance plasmid or by a chromosomal mutations. The location and diversity of the antibacterial resistance genes as well as their genetic characterization is important in identifying factors involved in the resistance. Mutations are also observed that modulate the gene expression at transcriptional or translational level of antibacterial resistant gene. Elaborate mechanisms that involve several metabolic pathways to achieve these resistances in the bacteria. In the course of time, the role of metabolism in the bacterial response to antibacterial has recently garnered interest because of a rapid rise in antibacterial resistance, which provides a clear link between metabolic function and cell viability and lack of novel targets to treat several infections.

\section{Discussion}

A large set of data documentation is available at present suggesting various mechanisms involved in antibacterial resistance ranging from drug-degrading enzymes to efflux pumps. Different levels of protections are afforded by these mechanisms in wide variety of bacteria and variation in metabolic impact of each resistance mechanisms [3]. Recent studies by independent groups, working with different bacteria suggest that efflux systems may be a first response to the antibacterial making possible for the cell to survive and acquire other, more stable resistance mechanisms certain bacteria [4]. Moreover, last decade has witnessed significant information that the efflux systems are one of the key contributions to the multidrug resistance in bacteria as a global problem. The outermembrane proteins (OMPs), the enzyme-coupled inner membrane proteins (IMPs) and the peripheral adoptive proteins (PAPs) are the basic composition of efflux pumps.

The lack of understanding of mechanisms of these pump assembly hindered the development of novel antibacterial, despite of advances in structural characterization of the pump proteins. These efflux pumps allows the microorganisms to regulate the internal environment by removing toxic substrates, including antimicrobial agents, metabolic and quorum sensing signal molecules by the number of components, the number of transmembrane-spanning regions, the enzyme source used by the pumps which are used to classify these pumps into six families: ABC, MFS, MATE, SMR, RND and DMT super families [5]. In certain wild-type strains of pathogenic bacteria, the genes encoding some MDR pumps are constitutively expressed 
which results in decreased antibacterial susceptibility. Presently, the drug resistant studies focus an importance on efflux genes induced resistance to antibacterial agents. The mechanisms that lead to increased expression of efflux pump genes may be due to mutations in local repressor genes, global regulatory genes, and promoter region of the transporter genes or insertion elements upstream of the transporter gene [6].

\section{Conclusion}

Understanding how the drug resistance is being evolved is representing a great opportunity and a change in the present biomedical research. Indeed, efforts are made to understand the evolution of antimicrobial resistance which could provide a window into how bacteria adopt to diverse environments which simultaneously elucidating novel drug targets to potentiate on current antibiotic arsenal. By considering the metabolic pathways that mediate genomic mutation by the bacterial efflux and two-component systems, the challenges of targeting these pathways can facilitate to explore new anti-evolutionary targets. These anti-evolutionary drugs will not reverse preexisting genetic resistance in nature or patients, rather than focusing on a pool of existing resistance. These agents could prevent or at least delay the de novo generation and acquisition of antimicrobial resistance in pathogens. By bridging the gap in our knowledge in antimicrobial resistance with the metabolism that the pathogen adopts and stochiometry of the pathways by computational biological and systems biology approaches tagged with molecular genetics will give a better understanding how the energy utilization in pathogens holds the promise of unmarking great opportunity to intervene.

\section{References}

1. Walsh C (2000) Molecular mechanisms that confer antibacterial drug resistance. Nature 406(6797): 775-781.

2. Mobeshery S, Azucen EF (2000) Bacterial Antibiotic resistance. In Encyclopedia of life sciences. Nature 406: 775-781.

3. Peng B, Su YB, Li H, Han Y, Guo C, Tian YM, et al. (2015) Exogenous alanine and/or glucose plus kanamycin kills antibiotic-resistant bacteria. Cell Metabolism 21(2): 249-261.

4. Blair JM, Smith HE, Ricci V, Lawler AJ, Thompson LJ, et al. (2015) Expression of homologous RND efflux pump genes is dependent upon AcrB expression: implications for efflux and virulence inhibitor design. Journal of Antimicrobial Chemotherapy 70(2): 424-431.

5. Bowman J, Ghosh PG (2014) A complex regulatory network controlling intrinsic multidrug resistance in Mycobacterium smegmetis. Molecular Microbiology 91(1): 121-134.

6. LJ Piddock, J Antimicrob Chemother (2001) Effect of hydrophobicity and molecular mass of the accumulation of fluoroquinolones by Staphylococcus aureus. Journal of Antimicrobial chemotherapy 47(3): 261-270.

7. Sun J, Deng Z, Yan A (2014) Bacterial multidrug efflux pumps: Mechanisms, physiology and pharmacological exploitations. Biochemical and Biophysical Research Communications 453(2): 254267. 